\title{
Prognostic Factors and Scoring Model for Survival in Metastatic Biliary Tract Cancer
}

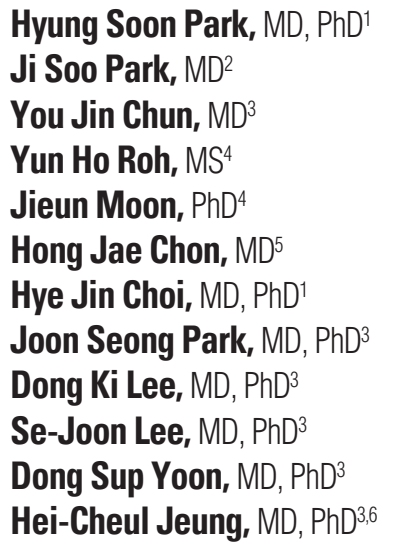

${ }^{1}$ Division of Medical Oncology, Yonsei Cancer Center, Yonsei University College of Medicine, Seoul, ${ }^{2}$ Cancer Prevention Center, Yonsei Cancer Center, Yonsei University College of Medicine, Seoul,

${ }^{3}$ Pancreatobiliary Cancer Clinic, Gangnam Severance Hospital, Yonsei University College of Medicine, Seoul, ${ }^{4}$ Biostatistics Collaboration Unit, Medical Research Center,

Yonsei University College of Medicine, Seoul, ${ }^{5}$ Division of Medical Oncology, Department of Internal Medicine, CHA Bundang Medical Center, CHA University, Seongnam, ${ }^{6}$ Songdang Institute for Cancer Research, Yonsei University College of Medicine, Seoul, Korea

\author{
Correspondence: Hei-Cheul Jeung, MD, PhD \\ Pancreatobiliary Cancer Clinic, \\ Gangnam Severance Hospital, \\ Yonsei University College of Medicine, \\ 211 Eonju-ro, Gangnam-gu, Seoul 06273, Korea \\ Tel: 82-2-2019-1242 \\ Fax: 82-2-3463-3882 \\ E-mail: jeunghc1123@yuhs.ac \\ Received November 14, 2016 \\ Accepted January 26, 2017 \\ Published Online February 6, 2017
}

\begin{abstract}
Purpose
Metastatic biliary tract cancer (mBTC) has a dismal prognosis. In this study, an independent dataset of patients with $\mathrm{mBTC}$ was used to implement and validate a routine clinico-laboratory parameter-based scoring model for risk group identification.
\end{abstract}

\section{Materials and Methods}

From September 2006 to February 2015, 482 patients with mBTC were assigned randomly (ratio, 7:3) into investigational $(n=340)$ and validation datasets $(n=142)$. The continuous variables were dichotomized using a normal range or the best cutoff values determined using the Contal and O'Quigley statistical methods. Following a Cox's proportional hazard model, the scoring model was derived by summing the rounded chi-square scores for the factors identified by multivariate analysis.

\section{Results}

The performance status (Eastern Cooperative Oncology Group 3-4), hypoalbuminemia $(<3.4 \mathrm{mg} / \mathrm{dL})$, carcinoembryonic antigen $(\geq 9 \mathrm{ng} / \mathrm{mL})$, neutrophil-to-lymphocyte ratio $(\geq 3.0)$, and carbohydrate antigen $19-9(\geq 120 \mathrm{U} / \mathrm{mL}$ ) were identified as independent prognosticators (Harrell's C index, 0.682; integrated area under the curve, 0.653). Survival was clearly correlated with the risk groups (low, intermediate, and high, 14.0, 7.3, and 2.3 months, respectively; $p<0.001$ ). The prognosis was also discriminative in the validation data set (median survival, 16.7, 7.5, and 1.9 months, respectively; $p<0.001$ ). Chemotherapy did not offer any survival benefits for high-risk patients.

\section{Conclusion}

These proposed prognostic criteria for $\mathrm{mBTC}$ can facilitate accurate patient risk stratification and treatment-related decision-making.

\section{Key words}

Biliary tract neoplasms, Prognosis, Scoring model, Survival, Validation, Drug therapy 


\section{Introduction}

Biliary tract cancer (BTC) comprises a heterogeneous group of tumors arising from the biliary tree-lining cells. These tumors are classified further as gallbladder cancer, ampullary cancer, or cholangiocarcinoma. The latter type is divided phenotypically into intrahepatic and ductal cholangiocarcinomas (hilar and non-hilar) to emphasize the distinctions between these clinically distinct cancers $[1,2]$. BTC is a relatively rare malignancy in Western populations; approximately 2,500 cases of cholangiocarcinoma and 5,000 cases of gallbladder cancer occur annually in the USA, yielding an average annual incidence of one case per 100,000 people. In contrast, multiple independent studies have documented a steady increase in the worldwide incidence of cholangiocarcinoma [3,4], and BTC is relatively common in Asian countries, including Korea, Japan, China, and India [5], with an approximate incidence of six cases per 100,000 people $[5,6]$. In 2013, 5,283 patients were newly diagnosed with BTC and 3,783 related deaths were reported in Korea, where BTC is the sixth-leading cause of cancer mortality.

Few treatment options are available for advanced BTC. Although a complete surgical resection remains the only curative treatment option, few patients are candidates for a potentially curative resection at the time of presentation. BTC is relatively chemo-/radio-resistant disease, and many patients are elderly and not healthy enough to tolerate aggressive treatment. Accordingly, these patients have dismal outcomes with a median survival of $<1$ year [7]. Currently, combination chemotherapy with cisplatin and gemcitabine (Gem/Cis) is considered the standard first-line palliative treatment, regardless of the primary tumor location, which is based on the results from the $\mathrm{ABC} 02$ trial [8]. On the other hand, the response rate to Gem/Cis is only $26 \%$, with a median overall survival (OS) of $\leq 11.7$ months. In other words, this regimen does not constitute an optimal treatment [8]. Therefore, it is essential to estimate the prognosis of a patient with metastatic BTC at the initial presentation, thus enabling patient individualization according to the risk and facilitating treatment optimization.

To date, several reports have addressed the prognostic parameters associated with BTC. For example, lymph node metastasis is a typical poor prognosticator for resectable BTC, and laboratory analyses indicated that a high C-reactive protein level and neutrophil-to-lymphocyte ratio (NLR) are correlated with survival in patients with resectable cancer or those receiving systemic chemotherapy. Despite this, few prognostic studies have addressed metastatic disease at diagnosis [9-12]. Of these, the majority targeted predictions after chemotherapy (particularly Gem/Cis). Although Eastern Cooperative Oncology Group performance status (ECOG
PS), NLR or absolute neutrophil counts, hemoglobin, white blood cell (WBC) counts, primary tumor location, and the number of metastatic diseases have been proposed, the significance of these factors is unclear. Most of these parameters were identified using small sample sizes with inadequate statistical power, and their roles have rarely been confirmed independently. Another problem is the risk of bias when the points of cutoff for the continuous variables are random, leading to the use of different cutoff points across studies, which imped a direct comparison between studies. Ultimately, in a real-world clinical setting, the question of "which patients to treat" should be answered prior to "which regimen to use," and that the answer to the former question requires the proposal of prognosticators that could discriminate patients who might and might not benefit from treatment.

Therefore, this paper presents the results of multivariate analysis of routinely evaluated clinico-laboratory parameters "at the time of initial diagnosis," to implement a scoring model that could effectively identify the risk groups, and validate the model in an independent dataset.

\section{Materials and Methods}

\section{Patients}

From September 2006 to February 2015, 623 patients were diagnosed with metastatic BTC at Gangnam Severance Hospital, Seoul, Korea. The diagnosis was made via a surgical excision, tissue biopsy, or cytology. The inclusion criteria for further analysis were as follows: (1) age > 18 years, (2) histologically confirmed diagnosis of BTC, (3) metastatic disease at the time of diagnosis or systemic recurrence after a curative resection, and (4) available electronic medical records (including treatment information).

The exclusion criteria were as follows: (1) widespread brain or leptomeningeal metastasis, (2) synchronous metastatic malignancies, (3) uncontrolled infection, active gastrointestinal bleeding, or other severe medical conditions, and (4) follow-up loss or transfer to another hospital prior to the decisions regarding treatment (including diagnosis only). Overall, 482 patients fulfilled these criteria and were further analyzed (Fig. 1). Our institutional review board approved this retrospective study and waived the requirement to obtain informed consent (3-2015-0318). 


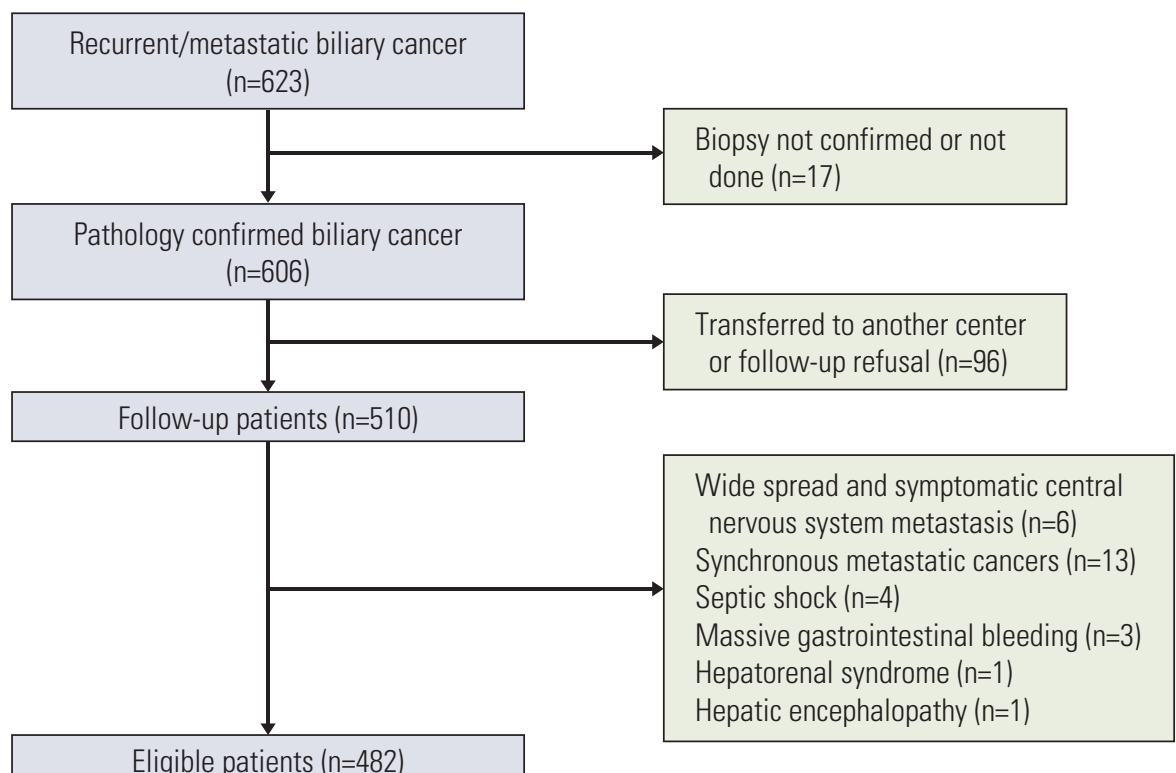

Fig. 1. Consort diagram of the enrolled patients.

\section{Data collection}

The following baseline data were recorded at the initial presentation: age, sex, disease status (recurrent/metastatic), primary tumor location (gallbladder, ampullary, or cholangiocarcinoma-intrahepatic or ductal), metastatic disease sites (liver, peritoneal carcinomatosis, lung, bone, lymph nodes, or others), ECOG PS, and body mass index (BMI). The hematological and blood chemistry values included the WBC and platelet counts as well as the hemoglobin, serum protein, albumin, blood urea nitrogen (BUN), aspartate aminotransferase (AST), alanine aminotransferase (ALT), total bilirubin, alkaline phosphatase (ALP), cholesterol, carcinoembryonic antigen (CEA), and carbohydrate antigen 19-9 (CA 19-9) levels. CEA was measured by a chemiluminescence immunoassay using UniCel DxI 800 immunoassay analyzer and CA 19-9 was measured by an electrochemiluminescence immunoassay using a Roche kit (Mannheim, Germany). The NLR was calculated by dividing the absolute neutrophil count by the absolute lymphocyte count, and the plateletto-lymphocyte ratio (PLR) was derived from the ratio of the platelets to lymphocytes.

Chest and abdomino-pelvic computed tomography (CT), radionuclide bone scan, and ${ }^{18} \mathrm{~F}$-fluorodeoxyglucose positron emission tomography were performed to evaluate the distant metastasis. Radiological detection of peritoneal carcinomatosis relied on the following findings, which were evaluated by an independent radiologist(s): thickening and nodular enhancement of peritoneal reflections, multiple soft tissue nodules, omental stranding and thickening (omental cake), small bowel mesentery stranding and distortion, and ascites, particularly if loculated. The presence of ascites was inferred from the evidence of malignant cell detection in a cytology examination or from suspicious fluid collection on CT or ultrasonography.

\section{Dataset allocation}

For the prognostic scoring model, the patients were assigned randomly into the investigation or validation dataset at a ratio of 7:3 using the primary tumor location stratification factor. The scoring model derived from the investigational dataset was validated internally using three methods, Harrell's C-index, integrated area under the curve (iAUC), and bootstrapping, followed by application to the independent validation dataset [13].

\section{Statistical analysis}

The hematological and blood chemistry values were initially recorded as continuous variables and later transformed to categorical variables according to the upper normal ranges (WBC, hemoglobin, platelet, serum protein, albumin, BUN, AST, ALT, bilirubin, ALP, and cholesterol) or the best cutoff point (CEA, CA 19-9, NLR, and PLR). The latter were determined using the Contal and $\mathrm{O}^{\prime}$ Quigley method, which cal- 
Table 1. Patient characteristics in the investigation and validation datasets

\begin{tabular}{|c|c|c|c|c|}
\hline Characteristic & $\begin{array}{c}\text { Total } \\
(n=482)\end{array}$ & $\begin{array}{c}\text { Investigation } \\
\text { dataset }(n=340)\end{array}$ & $\begin{array}{c}\text { Validation } \\
\text { dataset }(n=142)\end{array}$ & p-value \\
\hline \multicolumn{5}{|l|}{ Age (yr) } \\
\hline$<67$ & $235(48.8)$ & $163(47.9)$ & $72(50.7)$ & 0.58 \\
\hline$\geq 67$ & $247(51.2)$ & $177(52.1)$ & $70(49.3)$ & \\
\hline \multicolumn{5}{|l|}{ Sex } \\
\hline Male & $281(58.3)$ & $201(59.1)$ & $80(56.3)$ & 0.573 \\
\hline Female & $201(41.7)$ & $139(40.9)$ & $62(43.7)$ & \\
\hline \multicolumn{5}{|l|}{ Disease status } \\
\hline Recurrent & $158(32.8)$ & $117(34.4)$ & $41(28.9)$ & 0.238 \\
\hline Metastatic & $324(67.2)$ & $223(65.6)$ & $101(71.1)$ & \\
\hline \multicolumn{5}{|l|}{ ECOG } \\
\hline $0-2$ & $418(86.7)$ & $296(87.1)$ & $122(85.9)$ & 0.736 \\
\hline $3-4$ & $64(13.3)$ & $44(12.9)$ & $20(14.1)$ & \\
\hline \multicolumn{5}{|l|}{ Primary site } \\
\hline Intrahepatic CC & $123(25.5)$ & $87(25.6)$ & $36(25.4)$ & 1.00 \\
\hline Hilar CC & $79(16.4)$ & $56(16.5)$ & $23(16.2)$ & \\
\hline Non-hilar CC & $87(18.0)$ & $61(17.9)$ & $26(18.3)$ & \\
\hline Gallbladder & $146(30.3)$ & $103(30.3)$ & $43(30.3)$ & \\
\hline Ampullary & $47(9.8)$ & $33(9.7)$ & $14(9.9)$ & \\
\hline BMI & $22.8(20.6-25.0)$ & $22.7(20.4-24.9)$ & $23.2(21.0-25.1)$ & 0.223 \\
\hline \multicolumn{5}{|l|}{ Complete blood count } \\
\hline WBC count $(/ \mu \mathrm{L})$ & $7,090(5,525-9,615)$ & $7,070(5,555-9,650)$ & $7,130(5,328-9,563)$ & 0.881 \\
\hline Hemoglobin (g/dL) & $12.2(11.1-13.4)$ & $12.1(11.1-13.4)$ & $12.3(11.1-13.4)$ & 0.771 \\
\hline Platelet $\left(\times 10^{3} / \mu \mathrm{L}\right)$ & $243(189-319)$ & $241(186-314)$ & $250(200-326)$ & 0.398 \\
\hline NLR & $3.2(2.0-5.8)$ & $3.3(2.0-5.7)$ & $3.2(2.1-6.1)$ & 0.738 \\
\hline PLR & $171(122-251)$ & $165(120-234)$ & $181(123-275)$ & \\
\hline \multicolumn{5}{|l|}{ Blood chemistry profile } \\
\hline Protein $(\mathrm{g} / \mathrm{dL})$ & $6.9(6.5-7.4)$ & $6.9(6.5-7.4)$ & $7.0(6.5-7.4)$ & 0.804 \\
\hline Albumin (g/dL) & $3.9(3.4-4.3)$ & $3.9(3.4-4.3)$ & $3.9(3.4-4.3)$ & 0.775 \\
\hline BUN (mg/dL) & $14.7(11.5-19.2)$ & $14.8(11.6-19.3)$ & $14.4(11.3-18.9)$ & 0.671 \\
\hline AST (IU/L) & $36(22-75)$ & $37(23-81)$ & $35(22-68)$ & 0.433 \\
\hline $\operatorname{ALT}(\mathrm{IU} / \mathrm{L})$ & $30(17-74)$ & $30(17-72)$ & $29(18-76)$ & 0.850 \\
\hline Bilirubin (mg/dL) & $0.8(0.5-3.4)$ & $0.8(0.5-3.6)$ & $0.8(0.5-2.7)$ & 0.650 \\
\hline $\operatorname{ALP}(\mathrm{IU} / \mathrm{L})$ & $146(89-321)$ & $146(88-321)$ & $141(91-312)$ & 0.599 \\
\hline Cholesterol (mg/dL) & $167(134-198)$ & $168(134-194)$ & $165(132-202)$ & 0.900 \\
\hline \multicolumn{5}{|l|}{ Tumor marker } \\
\hline CEA (ng/mL) & $3.6(1.9-16.0)$ & $3.2(1.8-15.1)$ & $4.2(2.4-18.9)$ & 0.056 \\
\hline CA 19-9 (U/mL) & $160(26-1,472)$ & $166(25-1,587)$ & $145(27-1,298)$ & 0.567 \\
\hline \multicolumn{5}{|l|}{ Palliative Tx } \\
\hline No & $148(30.7)$ & $106(31.2)$ & $42(29.6)$ & 0.809 \\
\hline Gem/Cis & $168(34.9)$ & $120(35.3)$ & $48(33.8)$ & \\
\hline Others $^{\text {a) }}$ & $166(34.4)$ & $114(33.5)$ & $52(36.6)$ & \\
\hline
\end{tabular}

Values are presented as number (\%) or median (interquartile range). The Mann-Whitney $\mathrm{U}$ test was used for comparisons of continuous values. ECOG, Eastern Cooperative Oncology Group; CC, cholangiocarcinoma; BMI, body mass index; WBC, white blood cell; NLR, neutrophil-lymphocyte ratio; PLR, platelet-lymphocyte ratio; BUN, blood urea nitrogen; AST, aspartate aminotransferase; ALT, alanine aminotransferase; ALP, alkaline phosphatase; CEA, carcinoembryonic antigen; CA 19-9, carbohydrate antigen 19-9; Tx, treatment; Gem/Cis, combination chemotherapy with cisplatin and gemcitabine. ${ }^{a}$ Fluorouracil or gemcitabine-based (excluding Gem/Cis) chemotherapy. 
culates the maximum hazard ratio (HR) based on log-rank statistics and estimates the best cutoff value [14].

The OS was defined as the time from the date of diagnosis to death from any cause. Survival curves were generated using the Kaplan-Meier method and compared using the logrank test. Univariate analysis was performed to determine the association of the following prognostic factors with OS: age, sex, disease status, ECOG PS, primary site, BMI, WBC count, hemoglobin, platelet count, serum protein, albumin, BUN, AST, ALT, bilirubin, ALP, cholesterol, CEA, CA 19-9, NLR, and PLR. Subsequently, stepwise multivariate analysis based on Cox's proportional hazard model was performed using the significant factors in univariate analysis. HRs, 95\% confidence intervals (CIs), and chi-square scores were obtained for all regressions.

Using the investigation dataset, a risk scoring model was devised by summing the rounded chi-square scores of independent prognostic factors identified in the multivariate analysis. Finally, patients were grouped into three risk groups according to the risk scores: low risk, intermediate risk, and high risk. The discriminatory power of this scoring system was estimated using Harrell's c-index [15] and an iAUC. The latter was derived from time-dependent receiver operating characteristics curve analysis $[16,17]$. Finally, this

A
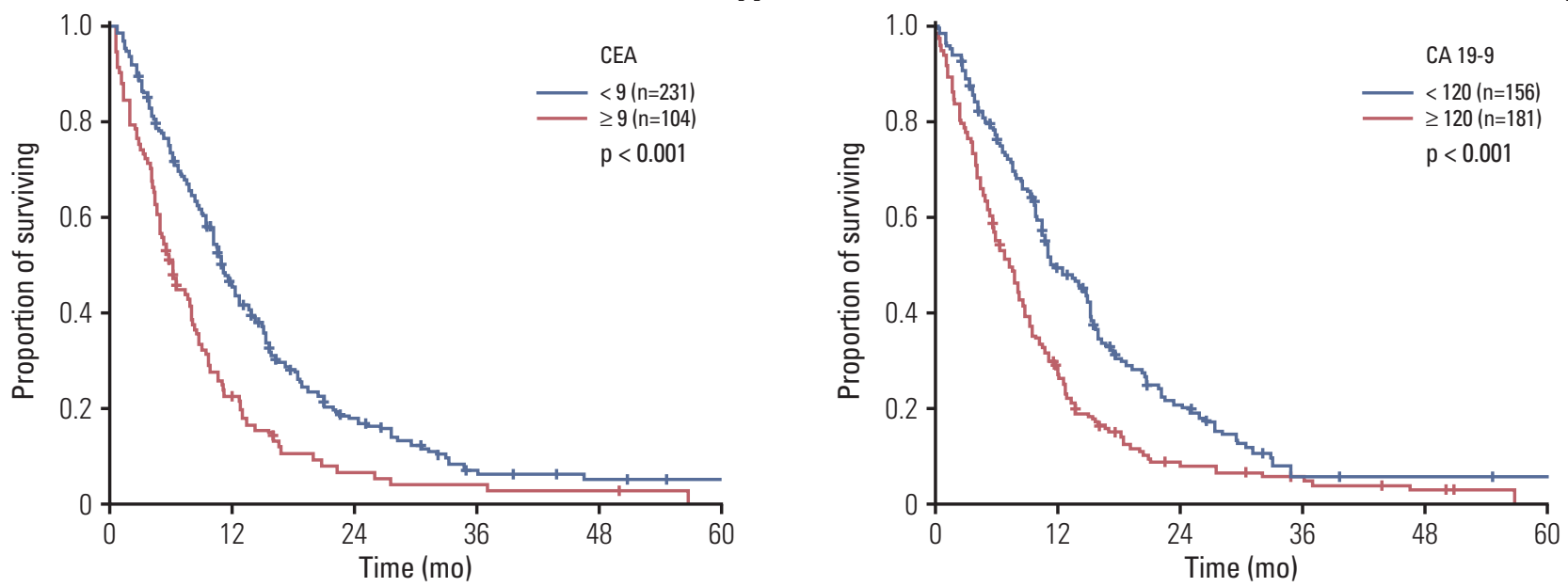

C
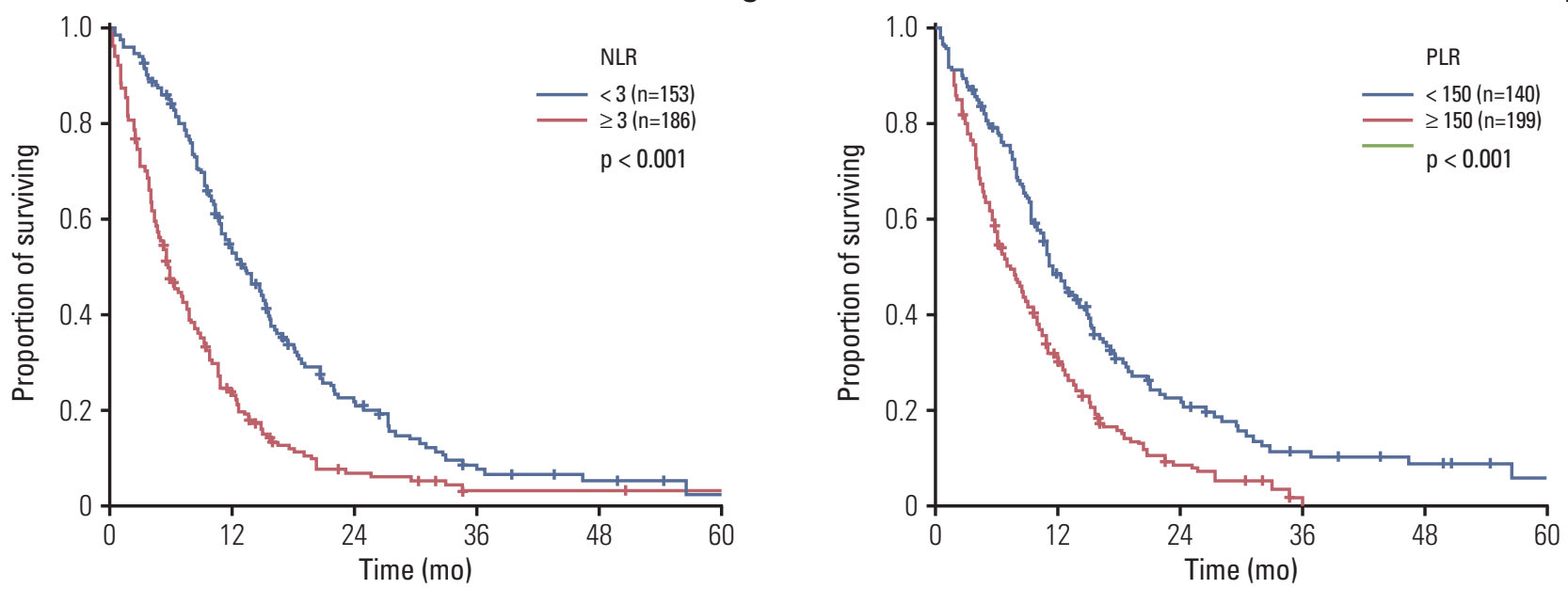

Fig. 2. Kaplan-Meier curves of overall survival for the investigation dataset ( $n=340)$ : carcinoembryonic antigen (CEA) (A), carbohydrate antigen 19-9 (CA 19-9) (B), neutrophil-to-lymphocyte ratio (NLR) (C), and platelet-to-lymphocyte ratio (PLR) (D). 
Table 2. Univariate analysis of the overall survival

\begin{tabular}{|c|c|c|c|c|c|c|}
\hline \multirow{2}{*}{ Characteristic } & \multicolumn{3}{|c|}{ Investigation dataset } & \multicolumn{3}{|c|}{ Validation dataset } \\
\hline & HR & $95 \% \mathrm{CI}$ & p-value & HR & $95 \%$ CI & p-value \\
\hline \multicolumn{7}{|l|}{ Age $(\mathrm{yr})$} \\
\hline$<67$ & 1 & & 0.02 & 1 & & 0.249 \\
\hline$\geq 67$ & 1.311 & $1.043-1.649$ & & 1.231 & $0.865-1.754$ & \\
\hline \multicolumn{7}{|l|}{ Sex } \\
\hline Male & 1 & & 0.515 & 1 & & 0.937 \\
\hline Female & 0.926 & $0.735-1.167$ & & 1.014 & $0.713-1.443$ & \\
\hline \multicolumn{7}{|l|}{ Disease status } \\
\hline Recurrent & 1 & & $<0.001$ & 1 & & 0.057 \\
\hline Metastatic & 1.584 & $1.24-2.023$ & & 1.462 & $0.989-2.161$ & \\
\hline \multicolumn{7}{|l|}{ ECOG } \\
\hline $0-2$ & 1 & & $<0.001$ & 1 & & $<0.001$ \\
\hline $3-4$ & 4.499 & $3.205-6.316$ & & 5.276 & $3.102-8.972$ & \\
\hline \multicolumn{7}{|l|}{ Primary site } \\
\hline Intrahepatic CC & 1 & & 0.045 & 1 & & 0.187 \\
\hline Hilar CC & 0.802 & $0.563-1.144$ & & 0.834 & $0.479-1.451$ & \\
\hline Non-hilar CC & 0.91 & $0.643-1.289$ & & 0.592 & $0.349-1.004$ & \\
\hline Gallbladder cancer & 0.94 & $0.692-1.276$ & & 0.895 & $0.56-1.431$ & \\
\hline Ampullary cancer & 0.514 & $0.331-0.8$ & & 0.519 & $0.255-1.057$ & \\
\hline \multicolumn{7}{|l|}{ BMI } \\
\hline$\geq 18.5$ & 1 & & 0.001 & 1 & & 0.024 \\
\hline$<18.5$ & 2 & $1.332-3.002$ & & 2.225 & $1.111-4.459$ & \\
\hline \multicolumn{7}{|l|}{ WBC count $(/ \mu \mathrm{L})$} \\
\hline Normal $(<10,800)$ & 1 & & $<0.001$ & 1 & & $<0.001$ \\
\hline Abnormal $(\geq 10,800)$ & 1.779 & $1.327-2.383$ & & 3.177 & 2.014-5.012 & \\
\hline \multicolumn{7}{|l|}{ Hemoglobin $(\mathrm{g} / \mathrm{dL})$} \\
\hline Normal ( $\geq 12)$ & 1 & & 0.005 & 1 & & 0.209 \\
\hline Abnormal $(<12)$ & 1.391 & $1.107-1.748$ & & 1.254 & $0.881-1.784$ & \\
\hline \multicolumn{7}{|l|}{ Platelet $\left(\times 10^{3} / \mu \mathrm{L}\right)$} \\
\hline Normal ( $\geq 150)$ & 1 & & 0.086 & 1 & & 0.814 \\
\hline Abnormal $(<150)$ & 1.375 & $0.955-1.98$ & & 0.935 & $0.535-1.634$ & \\
\hline \multicolumn{7}{|l|}{ Protein $(\mathrm{g} / \mathrm{dL})$} \\
\hline Normal $(\geq 6.9)$ & 1 & & 0.013 & 1 & & 0.006 \\
\hline Abnormal $(<6.9)$ & 1.337 & $1.064-1.681$ & & 1.635 & $1.149-2.325$ & \\
\hline \multicolumn{7}{|l|}{ Albumin (g/dL) } \\
\hline Normal ( $\geq 3.4)$ & 1 & & $<0.001$ & 1 & & 0.011 \\
\hline Abnormal $(<3.4)$ & 2.216 & $1.698-2.892$ & & 1.699 & $1.132-2.552$ & \\
\hline \multicolumn{7}{|l|}{ BUN (mg/dL) } \\
\hline Normal $(<23)$ & 1 & & 0.058 & 1 & & 0.01 \\
\hline Abnormal ( $\geq 23)$ & 1.401 & $0.988-1.988$ & & 1.956 & $1.178-3.25$ & \\
\hline \multicolumn{7}{|l|}{ AST (IU/L) } \\
\hline Normal $(<30)$ & 1 & & 0.151 & 1 & & 0.176 \\
\hline Abnormal $(\geq 30)$ & 1.187 & $0.94-1.501$ & & 1.279 & $0.895-1.826$ & \\
\hline \multicolumn{7}{|l|}{ ALT (IU/L) } \\
\hline Normal $(<33)$ & 1 & & 0.773 & 1 & & 0.237 \\
\hline Abnormal ( $\geq 33)$ & 1.034 & $0.823-1.299$ & & 1.239 & $0.869-1.768$ & \\
\hline \multicolumn{7}{|l|}{ Bilirubin (mg/dL) } \\
\hline Normal $(<1.2)$ & 1 & & 0.435 & 1 & & 0.198 \\
\hline Abnormal ( $\geq 1.2)$ & 1.099 & $0.867-1.394$ & & 1.277 & $0.88-1.853$ & \\
\hline
\end{tabular}


Table 2. Continued

\begin{tabular}{|c|c|c|c|c|c|c|}
\hline \multirow{2}{*}{ Characteristic } & \multicolumn{3}{|c|}{ Investigation dataset } & \multicolumn{3}{|c|}{ Validation dataset } \\
\hline & HR & $95 \% \mathrm{CI}$ & p-value & HR & $95 \% \mathrm{CI}$ & p-value \\
\hline \multicolumn{7}{|l|}{$\operatorname{ALP}(I U / L)$} \\
\hline Normal $(<123)$ & 1 & & 0.043 & 1 & & 0.043 \\
\hline Abnormal ( $\geq 123)$ & 1.27 & $1.007-1.6$ & & 1.439 & $1.011-2.05$ & \\
\hline \multicolumn{7}{|l|}{ Cholesterol (mg/dL) } \\
\hline Normal $(<139)$ & 1 & & 0.005 & 1 & & 0.022 \\
\hline Abnormal ( $\geq 139)$ & 1.437 & $1.114-1.854$ & & 1.586 & $1.068-2.353$ & \\
\hline \multicolumn{7}{|l|}{ CEA (ng/mL) } \\
\hline$<9$ & 1 & & $<0.001$ & 1 & & 0.016 \\
\hline$\geq 9$ & 1.812 & $1.414-2.321$ & & 1.604 & $1.094-2.353$ & \\
\hline \multicolumn{7}{|l|}{ CA $19-9(\mathrm{U} / \mathrm{mL})$} \\
\hline$<120$ & 1 & & $<0.001$ & 1 & & 0.441 \\
\hline$\geq 120$ & 1.668 & $1.323-2.104$ & & 1.149 & $0.807-1.637$ & \\
\hline \multicolumn{7}{|l|}{ NLR } \\
\hline$<3$ & 1 & & $<0.001$ & 1 & & $<0.001$ \\
\hline$\geq 3$ & 2.04 & $1.616-2.575$ & & 2.96 & $2.042-4.29$ & \\
\hline \multicolumn{7}{|l|}{ PLR } \\
\hline$<150$ & 1 & & $<0.001$ & 1 & & 0.057 \\
\hline$\geq 150$ & 1.719 & $1.355-2.181$ & & 1.43 & $0.99-2.066$ & \\
\hline \multicolumn{7}{|l|}{ Palliative Tx } \\
\hline No & 1 & & $<0.001$ & 1 & & $<0.001$ \\
\hline Gem/Cis & 0.484 & $0.364-0.643$ & & 0.284 & $0.180-0.448$ & \\
\hline Others ${ }^{\text {a) }}$ & 0.492 & $0.372-0.650$ & & 0.305 & $0.198-0.471$ & \\
\hline
\end{tabular}

HR, hazard ratio; CI, confidence interval; ECOG, Eastern Cooperative Oncology Group; CC, cholangiocarcinoma; BMI, body mass index; WBC, white blood cell; BUN, blood urea nitrogen; AST, aspartate aminotransferase; ALT, alanine aminotransferase; ALP, alkaline phosphatase; CEA, carcinoembryonic antigen; CA 19-9, carbohydrate antigen 19-9; NLR, neutrophillymphocyte ratio; PLR, platelet-lymphocyte ratio; Tx, treatment; Gem/Cis, combination chemotherapy with cisplatin and gemcitabine. ${ }^{\text {a)}}$ Fluorouracil or gemcitabine-based (excluding Gem/Cis) chemotherapy.

model was applied to the independent validation dataset.

All statistical analyses were performed using PASW Statistics ver. 18.0 (SPSS Inc., Chicago, IL), SAS ver 9.4 (SAS Institute Inc., Cary, NC), and R ver. 3.2.4 (Institute for Statistics and Mathematics, Vienna, Austria; http://www.R-project. org). A p-value of $<0.05$ was considered significant.

\section{Results}

\section{Baseline characteristics of investigational and validation datasets}

The investigational dataset included 340 patients with a median follow-up duration of 9.3 months (range, 0.3 to 78.1 months) from the initial admission. Table 1 lists the baseline demographics and clinico-laboratory parameters. The median age was 67 years, and a slight male predominance was observed (59.1\%). Regarding the primary tumor location, intrahepatic, hilar, and non-hilar cholangiocarcinoma affected $87(25.6 \%), 56(16.5 \%)$, and $61(17.9 \%)$ patients, respectively. Gallbladder and ampullary cancer was diagnosed in $103(30.3 \%)$ and $33(9.7 \%)$, respectively. The CEA, CA 19-9, NLR, and PLR cutoff values of $9 \mathrm{ng} / \mathrm{mL}, 120 \mathrm{U} / \mathrm{mL}$, 3.0, and 150, respectively, were determined using the Contal and $\mathrm{O}^{\prime}$ Quigley method.

Table 1 also lists the characteristics of the validation dataset. The distributions of the parameters investigated did not differ significantly between the investigation and validation datasets. 


\section{Assessment of the prognostic factors}

For the investigational dataset, the estimated median OS duration was 9.3 months (95\% CI, 8.2 to 10.4), and the CEA, CA 19-9, NLR, and PLR cutoff points were all found to be prognostic. In particular, the OS was significantly longer in patients with a low CEA (10.9 months vs. high 6.0 months, $p$ $<0.001$ ) and CA 19-9 levels (11.9 months vs. high 7.5 months, $\mathrm{p}<0.001)$, as well as those with a low NLR (13.5 months vs. high 5.9 months, $\mathrm{p}<0.001)$ and PLR $(11.4$ months vs. high 7.2 months, $\mathrm{p}<0.001$ ) (Fig. 2A-D). Regarding the tumor location, ampullary cancer was associated with a longer survival compared to other locations (14.1 months vs. gallbladder cancer 9.3 months; intrahepatic cholangiocarcinoma 8.4 months; non-hilar cholangiocarcinoma 8.7 months; hilar type 9.7 months).

The age, disease status, ECOG PS, primary tumor location, BMI, WBC count, hemoglobin, serum protein, albumin, ALP, and cholesterol had statistically significant associations with the OS in univariate analysis (Table 2). In multivariate analysis, including these 15 variables, a poor performance status (ECOG 3-4), hypoalbuminemia $(<3.4 \mathrm{mg} / \mathrm{dL})$, elevated CEA $(\geq 9 \mathrm{ng} / \mathrm{mL})$, high NLR $(\geq 3.0)$, and elevated CA 19-9 $(\geq 120$ $\mathrm{U} / \mathrm{mL}$ ) remained independent factors associated with a poor survival in forward stepwise analysis (Table 3).

\section{Inducement of prognostic model}

The above-listed five independent prognostic factors were used to derive a scoring system, in which scores of 0-8 were calculated by summing the rounded chi-square scores of these factors (Table 3): 2 (ECOG-PS $\geq 3$ ) +1 (albumin $<3.4$ $\mathrm{g} / \mathrm{dL})+1(\mathrm{CEA} \geq 9 \mathrm{ng} / \mathrm{mL})+1(\mathrm{CA} 19-9 \geq 120 \mathrm{U} / \mathrm{mL})+3(\mathrm{NLR}$ $\geq 7$ ). These scores were used to categorize the patients into three subgroups: (1) low-risk (score, 0-2; 147 [43.9\%]), (2) intermediate-risk (score, 3-5; 145 [43.3\%]), and (3) high-risk (score, 6-8; $43[12.6 \%]$ ) (see S1 Table for a summary of the baseline characteristics). The respective median OS durations were 14.0 months (95\% CI, 11.5 to 16.5), 7.3 months (95\% CI, 5.6 to 9.0 ), and 2.3 months (95\% CI, 0.1 to 4.6$)(\mathrm{p}<0.001$ ) (Fig. 3A), with corresponding 1-year survival rates of 55.9\%, $30.1 \%$, and $5.2 \%$, respectively. The internally validated Harrell's c-index was 0.682 (95\% CI, 0.663 to 0.701 ), and the scoring system iAUC was 0.654 (S2 Fig. A). In addition, bootstrapping to predict the 24-month survival revealed consistent results in the investigation dataset (S2 Fig. B).

For independent validation, the scoring model was applied to another dataset, and risk group categorization was performed, as described above, to yield $62(44.3 \%), 58$ (41.4\%), and $20(14.3 \%)$ patients in the low-risk, intermediate-risk, and high-risk categories, respectively; the corresponding median OS durations were 16.7 months (95\% CI, 14.9 to 18.5), 7.5 months (95\% CI, 3.8 to 11.2 ), and 1.9 months (95\% CI, 1.5 to 2.3$)(\mathrm{p}<0.001)$ (Fig. 3B). Harrell's c-index was $0.722(95 \%$ $\mathrm{CI}, 0.677$ to 0.767$)$, and the scoring system iAUC was 0.632

Table 3. Multivariate analysis of the overall survival and scoring index

\begin{tabular}{|c|c|c|c|c|c|}
\hline Factor & Chi-square score & HR & $95 \% \mathrm{CI}$ & p-value & Score \\
\hline \multicolumn{6}{|c|}{ ECOG performance status } \\
\hline $0-2$ & & 1.00 & & 0.001 & 0 \\
\hline $3-4$ & 10.5 & 2.10 & $1.34-3.28$ & & +2 \\
\hline \multicolumn{6}{|c|}{ Albumin $(\mathrm{g} / \mathrm{dL})$} \\
\hline$\geq 3.4$ & & 1.00 & & 0.017 & 0 \\
\hline$<3.4$ & 5.7 & 1.49 & $1.08-2.07$ & & +1 \\
\hline \multicolumn{6}{|c|}{ CEA $(\mathrm{ng} / \mathrm{mL})$} \\
\hline$<9$ & & 1.00 & & 0.033 & 0 \\
\hline$\geq 9$ & 4.5 & 1.35 & $1.02-1.78$ & & +1 \\
\hline \multicolumn{6}{|c|}{ CA 19-9 (U/mL) } \\
\hline$<120$ & & 1.00 & & 0.032 & 0 \\
\hline$\geq 120$ & 4.6 & 1.32 & $1.02-1.70$ & & +1 \\
\hline \multicolumn{6}{|l|}{ NLR } \\
\hline$<3$ & & 1.00 & & $<0.001$ & 0 \\
\hline$\geq 3$ & 14.5 & 1.64 & $1.27-2.11$ & & +3 \\
\hline
\end{tabular}

HR, hazard ratio; CI, confidence interval; ECOG, Eastern Cooperative Oncology Group; CEA, carcinoembryonic antigen; CA 19-9, carbohydrate antigen 19-9; NLR, neutrophil-lymphocyte ratio. 
A

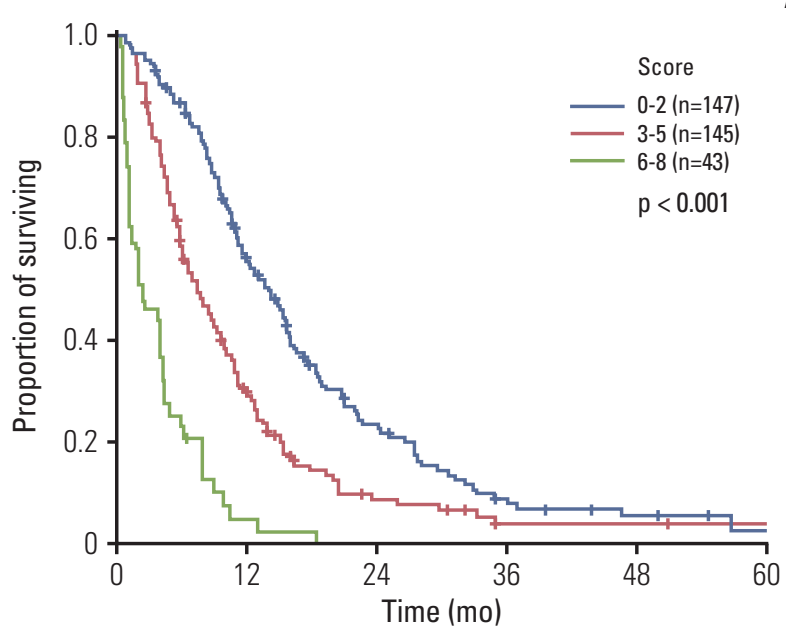

Fig. 3. Kaplan-Meier curves of overall survival according to the risk groups based on the prognostic prediction scores: low risk, 0-2; intermediate risk, 3-5; and high risk, 6-8; investigation (A) and validation datasets (B).

\section{(S2 Fig. A).}

Further internal validation was conducted using the primary site data from the investigation dataset. The scoring model was most reliable for non-hilar cholangiocarcinoma, and least reliable for intrahepatic cholangiocarcinoma and ampullary cancer (S3 Table).

\section{Associations with treatment outcomes}

In the investigational dataset, 234 patients $(68.8 \%)$ underwent palliative chemotherapy comprising Gem/Cis $(n=120$, $35.3 \%)$ or other regimens $(n=114,33.5 \%)$, including 5-fluorouracil (5-FU) or gemcitabine-based chemotherapy with/ without palliative radiotherapy (5-FU monotherapy $[\mathrm{n}=33$, 9.7\%], 5-FU / platinum $[\mathrm{n}=50,14.7 \%], 5-\mathrm{FU} /$ adriamycin $[\mathrm{n}=5$, $1.5 \%$ ], 5-FU/gemcitabine $[\mathrm{n}=5,1.5 \%$, gemcitabine monotherapy $[n=13,3.8 \%]$, and gemcitabine/oxaliplatin $[n=8,2.4 \%])$. On the other hand, 98 patients $(28.8 \%)$ received the best supportive care (BSC) and eight $(2.4 \%)$ received palliative radiotherapy for bone metastasis $(n=3,0.9 \%)$ or local control $(n=5$, $1.5 \%$ ). Patients receiving Gem/Cis or other regimens had a significantly longer survival than those who received BSC (median OS, 11.0 months vs. 11.3 months vs. 4.0 months, respectively; $\mathrm{p}<0.001)$.

One hundred eighteen $(80.3 \%)$ and $96(66.3 \%)$ patients in the low- and intermediate-risk groups received chemotherapy, whereas only 18 high-risk patients $(41.9 \%)$ consented to chemotherapy. In the former group, old age, hypoalbuminemia, leukocytosis, and anemia were the determining factors regarding the selection of $\mathrm{BSC}$, whereas a young age was the

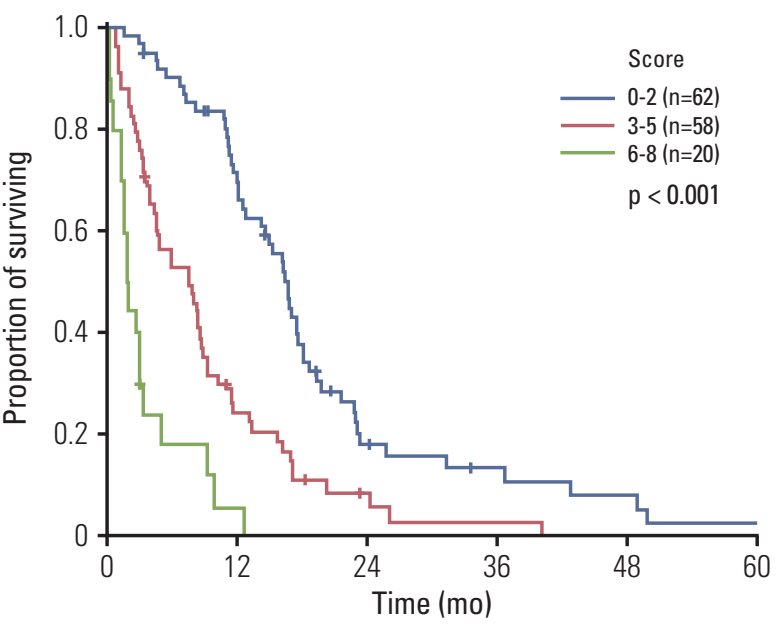

most important predictor of a preference for chemotherapy in the high-risk group. Palliative chemotherapy (Gem/Cis or other regimens) yielded a significant survival benefit in the low- and intermediate-risk patients (median OS, 11.4 months vs. 12.1 months vs. 4.8 months; $\mathrm{p}<0.001$ ) (Fig. 4A), whereas the high-risk patients did not receive such benefits (median OS, 4.3 months vs. 3.8 months vs. 1.1 months; $\mathrm{p}=0.105$ ) (Fig. 4B). Similar results were observed in the validation dataset (Fig. 4C and D).

\section{Discussion}

To the best of the authors' knowledge, this is the first study to implement a prognostic scoring model for patients with metastatic BTC at the time of the initial disease presentation. This model might better indicate which patients would benefit from treatment because given the relative rarity of BTC, most previous studies enrolled subjects irrespective of the primary location, disease extent (local or distant metastasis), and disease status (recurrent or unresectable). Moreover, many patients with BTC are quite malnourished and have a poor general state due to the dual effects of malignancy and obstructive jaundice. Because of this population heterogeneity, identification of the prognostic factors is crucial for clinical trial design and stratification factor selection.

Most studies included patients who received palliative chemotherapy, whereas the proportions of patients receiving 
A
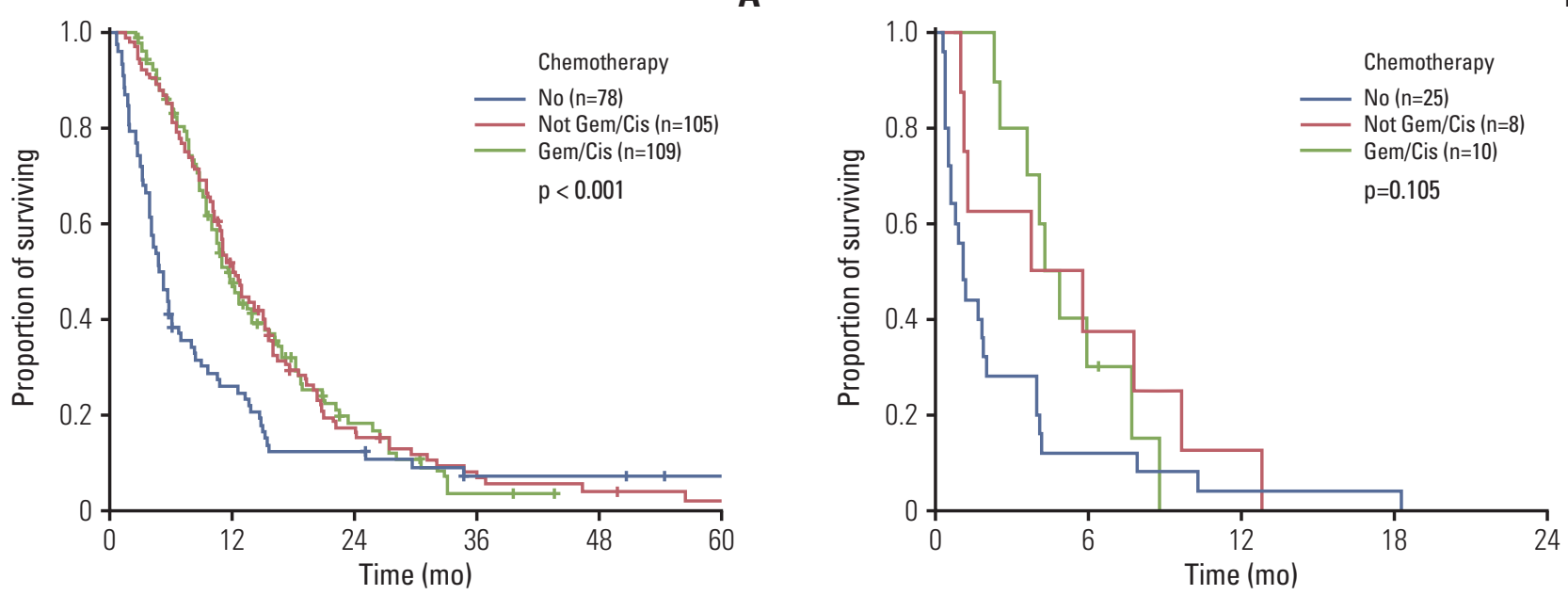

C
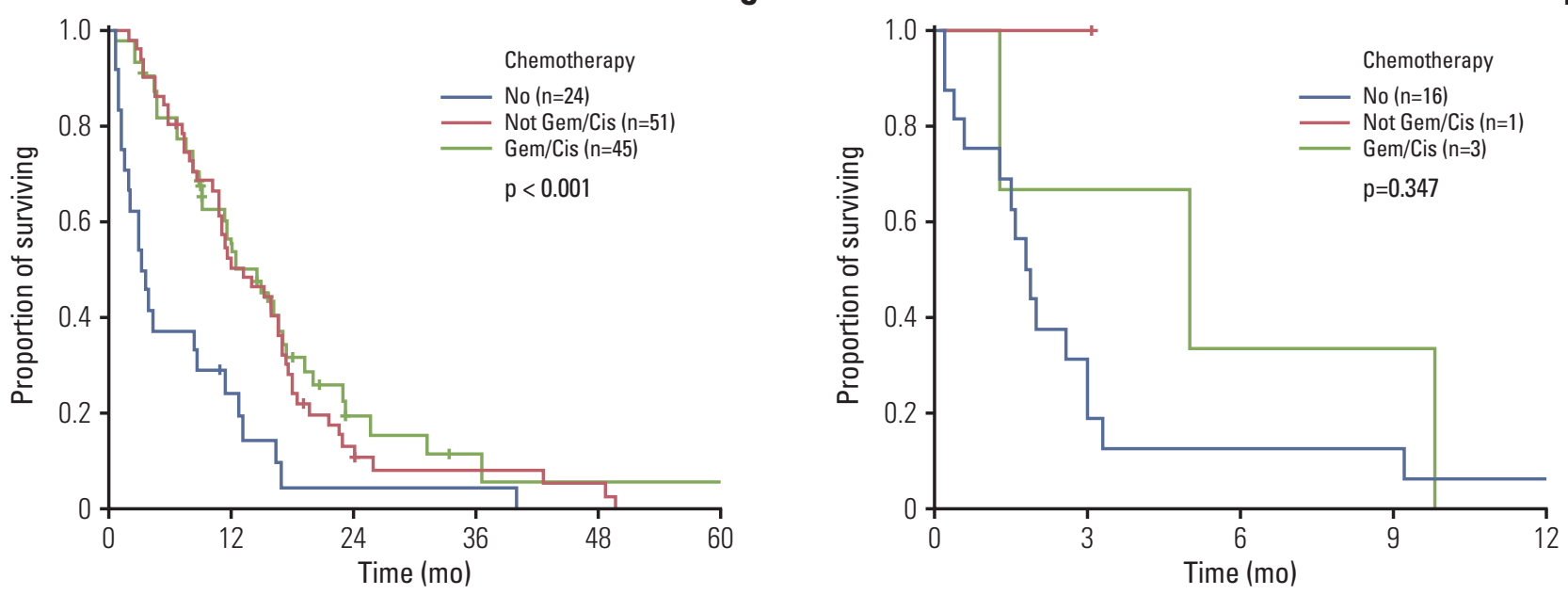

Fig. 4. Kaplan-Meier curves of the overall survival according to palliative chemotherapy use in the investigation and validation datasets: low-intermediate-risk (A, C) and high-risk (B, D), respectively. Gem/Cis, combination chemotherapy with cisplatin and gemcitabine.

active chemotherapy was not high, leading to a small number of study samples and low statistical power. This study showed that one clinical parameter (ECOG PS) and four laboratory parameters (serum albumin, CEA, CA 19-9, and NLR) affected the patient survival. ECOG-PS is a well-established, robust prognosticator in a variety of cancers, including BTC $[9,11]$. Although hypoalbuminemia is not a widely accepted BTC parameter, it is a key component of the modified Glasgow Prognostic Score, which can predict the cancer survival independently of the tumor site $[12,18]$. The pretreatment serum albumin levels provide useful prognostic information [19] because albumin synthesis reflects malnu- trition and tumor-related inflammation through the production of cytokines, such as interleukin 6 [19]. A third of these patients were identified as nutritionally at-risk upon admission, according to the Malnutrition Universal Screening Tool (MUST), leading to the initiation of nutritional care plans [20].

These results are comparable to those of McNamara et al. [9], who reported the associations of a poor ECOG PS, low albumin level, and high NLR with reduced survival in patients with BTC. On the other hand, that study included a heterogeneous dataset that incorporated all the disease stages (I-IV) and a wide range of treatments (curative surgery 
to palliative therapy), and found that the primary location, stage, lack of surgery, and anemia were also associated with survival. In contrast, McNamara et al. [9] identified ECOG PS as the most significant prognostic factor; the NLR had the biggest impact on survival in this study. NLR, a marker of host inflammation, is a prognostic factor in several cancers. The prognostic significance of NLR might be related to reduced immunocompetence or neutrophilia, which would contribute to a tumor-promoting microenvironment characterized by suppressed host immune surveillance. Furthermore, its significance might be attributable to either a predisposition to chronic inflammation, a known risk factor for cancer development, or cancer-associated inflammation, which was also reported to be a key determinant of disease progression in many cancers [9].

Previous clinical and population-based studies examined the effects of various inflammatory factors on BTC development [21-24]. In metastatic BTC, the large cancer cell burden may lead to paraneoplastic production of myeloid growth factors (e.g., granulocyte colony-stimulating factor), and consequently, leukocytosis. Lymphocytes play a critical role in the tumor defense by inducing immune-mediated cytotoxic cell death; accordingly, lymphopenia in a metastatic setting reflects the reduced immunocompetence and the effects of lympholytic cytokines produced by cancer or host cells. Recent findings suggest that in cancer patients, lymphopenia might arise from tumor-mediated lymphocyte destruction and / or altered lymphocyte homeostasis [25]. Cancer-associated neutrophils also produce vascular endothelial growth factor, matrix metallopeptidase-9, reactive oxygen species, and arginase 1, which encourage tumor development and inhibit the functions of cytotoxic lymphocytes [26,27]. Interestingly, a high PLR was not found to be significant in this multivariate analysis, possibly because of the significant link between NLR and PLR (correlation co-efficient value, 0.446; $\mathrm{p}<0.001$ ).

The markers, CEA and CA 19-9, are non-specific because their expression may increase in response to extrahepatic obstruction from any cause, and their cutoff values are more obscure in the context of metastatic BTC. Previous studies usually adopted the median values or normal ranges to dichotomize these continuous variables; however, some of the percentiles and averages were arbitrary and might not reveal the true prognostic value of these variables [28]. Accordingly, the Contal and O'Quigley method, which selects a cutoff point that maximizes the model likelihood only after evaluating all possible cutoffs, was implemented and useful cutoff values of $9 \mathrm{ng} / \mathrm{mL}$ for CEA and $120 \mathrm{U} / \mathrm{mL}$ for CA 19-9 were estimated in patients with metastatic BTC. On the other hand, this study focused only on the dichotomization of a continuous covariate, assuming that such dichotomization would be possible from a biological point of view; however, more than one cutoff may exist in reality. Furthermore, an ideal cutoff search would work within the framework of a multiple regression model to eliminate the potential influences of other prognostic factors. Nevertheless, this study provides insights into some of the data and outcome-oriented cutoff determination methods.

The retrospective study design introduced some limitations with regard to interpreting the effects of the different treatment regimens used in this study. Gem/Cis has been available and accepted widely in Korea since 2011. Prior to that, various monotherapies and doublet regimens that included 5-FU, gemcitabine, and platinum agents were used. In addition, $>30 \%$ of patients failed to start first-line chemotherapy. In the low-risk group, $20 \%$ of patients refused chemotherapy because of old age, poor nutritional status, and combined inflammation; in contrast, $>40 \%$ of patients in the high-risk group attributed their decision in favor of chemotherapy to their young age. This scoring system is expected to allow more sophisticated treatment decisions. For example, patients with a score of $\leq 2$ could expect their survival to be extended by up to 16 months with active chemotherapy (Gem/Cis or other regimens), and these patients would be candidates for more aggressive regimens or clinical trials. On the other hand, high-risk patients (score $\geq 6$ ) are unlikely to benefit from chemotherapy. Nevertheless, it would be imprudent to conclude that chemotherapy is contraindicated for this group because the survival curves are still separated between chemotherapy and best supportive group, even though statistical significance was not reached. This may be related to the lack of power, in which only small number of patients was included for analysis. Alternatively, monotherapy could be a rational option, even though a prospective evaluation will be needed.

Regarding the intermediate group, patients treated with chemotherapy had a median survival of 9.9 months, suggesting that the general survival benefit of chemotherapy has not been established clearly; still, chemotherapy yielded a significant difference in survival (9.9 months vs. without 4.2 months; $p=0.006$, log-rank test). Therefore, chemotherapy is recommended for patients with a good clinical condition. On the other hand, patients with general deterioration (ECOG 2) or insufficient biliary decompression will receive only small benefits from chemotherapy, and its indications should be considered carefully. Overall, these findings suggest that the proposed scoring system could facilitate the selection of the "right" patients for the "right" treatment plan.

In this study, multiple internal validation analyses were performed. The Harrell's c-index (0.682), iAUC (0.654), and bootstrapping showed that the proposed model possesses meaningful risk-based patient discrimination power [29]. Given the rarity of BTC, validation studies involving independent datasets are uncommon; internal validation metho- 
dologies were considered to be insufficiently credible and it is believed that the inclusion of an independent test set validation is the strength of the present study. Validation using an independent set confirmed that this scoring system clearly divided patients according to risk. On the other hand, there was another limitation. Although the investigation and validation datasets comprised independent populations, all patients were treated at a single center. Therefore, the proposed scoring model needs to be assessed in an external validation dataset to improve the robustness.

In conclusion, a set of prognostic criteria were proposed to determine the survival outcomes of patients with metastatic BTC, which were validated independently. Specifically, this system suggests cutoff values for the ECOG PS, albumin, CEA, CA 19-9, and NLR, which were identified as independent prognostic factors. In addition, the prognostic scoring system provides a rationale for the risk stratification of patients with metastatic BTC. Clinicians should find that this system facilitates treatment-related decision making.

\section{Electronic Supplementary Material}

Supplementary materials are available at Cancer Research and Treatment website (http://www.e-crt.org).

\section{Conflicts of Interest}

Conflict of interest relevant to this article was not reported.

\section{Acknowledgments}

This study was supported by a grant from the National R\&D Program for Cancer Control, Ministry of Health \& Welfare, Republic of Korea (to H.C. Jeung, No. 1420060).

\section{References}

1. de Groen PC, Gores GJ, LaRusso NF, Gunderson LL, Nagorney DM. Biliary tract cancers. N Engl J Med. 1999;341:1368-78.

2. Patel T. Cholangiocarcinoma: controversies and challenges. Nat Rev Gastroenterol Hepatol. 2011;8:189-200.

3. Khan SA, Taylor-Robinson SD, Toledano MB, Beck A, Elliott $\mathrm{P}$, Thomas HC. Changing international trends in mortality rates for liver, biliary and pancreatic tumours. J Hepatol. 2002;37:806-13.

4. Welzel TM, McGlynn KA, Hsing AW, O'Brien TR, Pfeiffer RM. Impact of classification of hilar cholangiocarcinomas (Klatskin tumors) on the incidence of intra- and extrahepatic cholangiocarcinoma in the United States. J Natl Cancer Inst. 2006;98: 873-5.

5. Randi G, Malvezzi M, Levi F, Ferlay J, Negri E, Franceschi S, et al. Epidemiology of biliary tract cancers: an update. Ann Oncol. 2009;20:146-59.

6. Oh CM, Won YJ, Jung KW, Kong HJ, Cho H, Lee JK, et al. Cancer statistics in Korea: incidence, mortality, survival, and prevalence in 2013. Cancer Res Treat. 2016;48:436-50.

7. Siegel RL, Miller KD, Jemal A. Cancer statistics, 2016. CA Cancer J Clin. 2016;66:7-30.

8. Valle J, Wasan H, Palmer DH, Cunningham D, Anthoney A, Maraveyas A, et al. Cisplatin plus gemcitabine versus gemcitabine for biliary tract cancer. N Engl J Med. 2010;362: 1273-81.

9. McNamara MG, Templeton AJ, Maganti M, Walter T, Horgan AM, McKeever L, et al. Neutrophil/lymphocyte ratio as a prognostic factor in biliary tract cancer. Eur J Cancer. 2014;50: 1581-9.
10. Peixoto RD, Renouf D, Lim H. A population based analysis of prognostic factors in advanced biliary tract cancer. J Gastrointest Oncol. 2014;5:428-32.

11. Bridgewater J, Lopes A, Wasan H, Malka D, Jensen L, Okusaka T, et al. Prognostic factors for progression-free and overall survival in advanced biliary tract cancer. Ann Oncol. 2016;27: $134-40$.

12. Iwaku A, Kinoshita A, Onoda H, Fushiya N, Nishino H, Matsushima M, et al. The Glasgow Prognostic Score accurately predicts survival in patients with biliary tract cancer not indicated for surgical resection. Med Oncol. 2014;31:787.

13. Steyerberg EW, Harrell FE Jr, Borsboom GJ, Eijkemans MJ, Vergouwe Y, Habbema JD. Internal validation of predictive models: efficiency of some procedures for logistic regression analysis. J Clin Epidemiol. 2001;54:774-81.

14. Contal C, O'Quigley J. An application of changepoint methods in studying the effect of age on survival in breast cancer. Comput Stat Data Anal. 1999;30:253-70.

15. Harrell FE Jr, Lee KL, Matchar DB, Reichert TA. Regression models for prognostic prediction: advantages, problems, and suggested solutions. Cancer Treat Rep. 1985;69:1071-7.

16. Heagerty PJ, Zheng Y. Survival model predictive accuracy and ROC curves. Biometrics. 2005;61:92-105.

17. Chambless LE, Diao G. Estimation of time-dependent area under the ROC curve for long-term risk prediction. Stat Med. 2006;25:3474-86

18. Proctor MJ, Talwar D, Balmar SM, O'Reilly DS, Foulis AK, Horgan PG, et al. The relationship between the presence and site of cancer, an inflammation-based prognostic score and 
biochemical parameters. Initial results of the Glasgow Inflammation Outcome Study. Br J Cancer. 2010;103:870-6.

19. Gupta D, Lis CG. Pretreatment serum albumin as a predictor of cancer survival: a systematic review of the epidemiological literature. Nutr J. 2010;9:69.

20. Kondrup J, Allison SP, Elia M, Vellas B, Plauth M; Educational and Clinical Practice Committee, European Society of Parenteral and Enteral Nutrition (ESPEN). ESPEN guidelines for nutrition screening 2002. Clin Nutr. 2003;22:415-21.

21. Hsing AW, Gao YT, Han TQ, Rashid A, Sakoda LC, Wang BS, et al. Gallstones and the risk of biliary tract cancer: a population-based study in China. Br J Cancer. 2007;97:1577-82.

22. Hsing AW, Gao YT, McGlynn KA, Niwa S, Zhang M, Han TQ et al. Biliary tract cancer and stones in relation to chronic liver conditions: a population-based study in Shanghai, China. Int J Cancer. 2007;120:1981-5.

23. Hsing AW, Zhang M, Rashid A, McGlynn KA, Wang BS, Niwa $S$, et al. Hepatitis $B$ and $C$ virus infection and the risk of biliary tract cancer: a population-based study in China. Int J Cancer. 2008;122:1849-53.

24. Liu E, Sakoda LC, Gao YT, Rashid A, Shen MC, Wang BS, et al. Aspirin use and risk of biliary tract cancer: a populationbased study in Shanghai, China. Cancer Epidemiol Biomarkers Prev. 2005;14:1315-8.

25. Saito T, Kuss I, Dworacki G, Gooding W, Johnson JT, Whiteside TL. Spontaneous ex vivo apoptosis of peripheral blood mononuclear cells in patients with head and neck cancer. Clin Cancer Res. 1999;5:1263-73.

26. Powell DR, Huttenlocher A. Neutrophils in the tumor microenvironment. Trends Immunol. 2016;37:41-52.

27. Spolverato G, Maqsood H, Kim Y, Margonis G, Luo T, Ejaz A, et al. Neutrophil-lymphocyte and platelet-lymphocyte ratio in patients after resection for hepato-pancreatico-biliary malignancies. J Surg Oncol. 2015;111:868-74.

28. Mazumdar M, Glassman JR. Categorizing a prognostic variable: review of methods, code for easy implementation and applications to decision-making about cancer treatments. Stat Med. 2000;19:113-32.

29. Keam B, Kim DW, Park JH, Lee JO, Kim TM, Lee SH, et al. Nomogram predicting clinical outcomes in non-small cell lung cancer patients treated with epidermal growth factor receptor tyrosine kinase inhibitors. Cancer Res Treat. 2014;46:323-30. 